\title{
DIVERGENT CANCER PATHWAYS FOR EARLY-ONSET AND LATE-ONSET CUTANEOUS MALIGNANT MELANOMA
}

\author{
William F. Anderson, MD, MPH, \\ Biostatistics Branch (BB), DHHS/NIH/NCl/Division of Cancer Epidemiology and Genetics (DCEG), \\ EPS, Room 8036, 6120 Executive Blvd, Bethesda, MD 20892-7244, Phone: 301 594-9125, Fax: \\ 301 402-0081, Email: wanderso@mail.nih.gov \\ Ruth M. Pfeiffer, PhD, \\ DHHS/NIH/NCI/DCEG/BB, Email: Pfeiffer@mail.nih.gov
}

Margaret A. Tucker, MD, and

DHHS/NIH/NCl/DCEG/GEB, Email: tuckerp@mail.nih.gov

Philip S. Rosenberg, PhD

DHHS/NIH/NCl/DCEG/BB, Email: rosenbep@mail.nih.gov

\begin{abstract}
Background-Emerging data suggest that cutaneous malignant melanomas (CMM) may arise through divergent cancer pathways, linked to intermittent versus accumulated sun exposure. However, numerous questions remain regarding the timing and/or age of exposure.

Methods-We, therefore, systematically examined the effect of aging upon CMM incidence in the Surveillance, Epidemiology, and End Results program of the National Cancer Institute. Standard descriptive epidemiology was supplemented with mathematical models. The impact of advancing age upon CMM incidence was assessed by gender, histopathological classification (superficial spreading melanoma (SSM) or lentigo maligna melanoma (LMM)), and anatomic site (face, head, and neck (FHN) or lower extremity (LE)).
\end{abstract}

Results-Gender, histopathology, and anatomic site were age-specific effect modifiers for CMM, showing divergent (bimodal) early- and late-onset cancer pathways. Early-onset melanomas were predominantly associated with female gender, SSM, and LE. Late-onset melanomas were correlated with male gender, LMM, and FHN. Early- and late-onset melanoma populations were confirmed with age-period-cohort models that were adjusted for period and cohort effects. Two-component mixture models also fit the data better than a single cancer population.

Conclusions-These results are consistent with a divergent and age-dependent solar hypothesis for CMM. Early-onset melanomas may represent gene-sun exposure interactions occurring early (and/or intermittently) in life among susceptible individuals. Late-onset melanomas possibly reflect accumulated lifelong sun exposure in comparatively less susceptible individuals. Future analytical studies should be adequately powered to account for this age-dependent effect modification for acknowledged (gender, histopathology, and anatomic site) as well as suspected melanoma risk factors such as constituent genetic variants.

\section{Keywords}

Superficial spreading melanoma; Lentigo maligna melanoma; Poisson regression models; two component mixture models; age period cohort models; SEER 


\section{INTRODUCTION}

Solar irradiation is generally considered to be a direct risk factor for cutaneous malignant melanomas (CMMs), although the relationship between sun exposure and melanoma risk is complex. ${ }^{1,2}$ For example, in North America, increased CMM incidence is positively associated with a North-South gradient in ultraviolet (UV) light exposure and higher levels of UV exposure as measured by climatic, atmospheric, and recreational exposure. ${ }^{3,4} \mathrm{CMM}$ incidence also is generally higher among persons with fair skin. On the other hand, some observations suggest an indirect relationship between sun exposure and CMM. That is, CMMs are more common among indoor than outdoor workers and in intermittently sun exposed skin. ${ }^{5,6}$ Earlyonset melanomas are also more affected by intermittent than continuous sun exposure. ${ }^{7}$

These observations imply that CMMs might arise through at least two different cancer pathways, one of which is associated with intermittent and the other with chronic sun exposure. $8,{ }^{9}$ However, many uncertainties remain regarding the dual effects of sun exposure and melanoma risk such as the timing and/or age of exposure. ${ }^{10}$ Indeed, unlike many epithelial tumors with peak incidence rates at older ages, CMMs commonly arise in younger persons. Therefore, to assess whether descriptive data are consistent with an age-dependent twopathway CMM model, we examined the impact of age on CMM incidence and the variability of age affects with other factors such as gender, histopathological classification, and anatomic body site. We used the National Cancer Institute's Surveillance, Epidemiology, and End Results program to obtain large numbers of CMM cases with population-based incidence rates.

We supplemented standard descriptive epidemiology with mathematical models. Poisson regression models provided formal significance tests of age interactions (or effect modifications) by gender, histopathological classification, or body site; statistical interaction is consistent with biological interactions due to cancer heterogeneity. ${ }^{11}$ Two component mixture models identified heterogeneity in age distributions at diagnosis by determining whether two or more melanoma populations fit the data better than a single cancer population. Age-period-cohort (APC) models assessed "fitted" age-specific incidence rates, adjusted for calendar-period (i.e. screening, case ascertainment, etc) and/or birth-cohort (i.e. risk factor and exposure) effects. ${ }^{12}$ Variations in the shape of the APC fitted age-at-onset curves across different CMM populations with differential exposure patterns suggest etiological heterogeneity.

\section{MATERIALS AND METHODS}

We used the nine Registries Database of the National Cancer Institute's Surveillance, Epidemiology, and End Results (SEER 9) Program to examine cutaneous invasive malignant melanoma, diagnosed during the years 1975-2004. ${ }^{13}$ In-situ lesions were excluded from analysis. SEER's 9 tumor registries were located in Atlanta, Connecticut, Detroit, Hawaii, Iowa, New Mexico, San Francisco-Oakland, Seattle-Puget Sound, and Utah; and covered approximately $10 \%$ of the United States population. We analyzed data for all races combined, although Whites accounted for $96.4 \%$ ( $\mathrm{n}=93,378$ of 96,900) of all melanoma cases.

\section{Demographic and tumor characteristics}

These included gender, age at diagnosis, race, histopathologic classification, anatomic body site, and tumor thickness in millimeters (i.e., Breslow's measurement). Descriptive data also were stratified by three 10-year time-periods (1975-84, 1985-94, and 1995-2004). SEER collected all case-related data for the entire study period except for tumor thickness, which was recorded from 1988 forward (1988+). 
Histopathologic classification and anatomic body site were defined using the International Classification of Diseases for Oncology-3 ${ }^{\text {rd }}$ edition (ICD-O-3).${ }^{14}$ Histopathologic classifications included superficial spreading melanoma (SSM, ICD-0-3 8743), nodular melanoma (NM, ICD-O-3 8721), lentigo maligna melanoma (LMM, ICD-O-3 8742), acral melanoma (ACM, ICD-O-3 8744), unclassified and/or not otherwise specified melanoma (UCM, all remaining ICD-O-3 codes), and unknown. Anatomic body sites were face, head, and neck (FHN, topography 440-444), upper extremity (UE, topography 446), trunk (topography 445, including back, abdomen, and chest), lower extremity (topography LE, 447), with all "other or unknown" body sites combined into a single category. Anatomic body site was further categorized into chronically (FHN and UE) versus intermittently (trunk and LE) sun exposed skin.

\section{Statistical methods}

Age-adjusted (2000 United States standard population) incidence rates with standard error (SE) were obtained using SEER*Stat 6.3.6 and expressed per 100,000 person-years (or persons per year). Incidence rate ratios (IRR) were calculated as relative risks, where a given characteristic was compared to a referent characteristic with an assigned IRR of 1.0. IRRs were tested for statistical significance at the $95 \%$ confidence level. All statistical tests were two-sided.

Age-adjusted incidence trends by gender were plotted on a log-linear scale by six 5-year timeperiods (1975-79, 1980-84, 1985-89, 1990-94, 1995-99, and 2000-04). Cross-sectional agespecific incidence rates by gender were plotted on a log-linear scale by eighteen age categories; i.e., seventeen 5-year age groups $(0-4,5-9, \ldots 80-84)$ and one age group with all persons age 85 years or older. Poisson regression models were used to examine age interaction by gender. We tested for age interaction by gender (modeled as a regression spline) with use of a likelihood ratio test. ${ }^{12}$ Statistically significant age interactions could be quantitative (non-crossover) or qualitative (crossover). Qualitative age interactions, in particular, were strong surrogates for etiological heterogeneity. ${ }^{11,15}$

Probability density functions by gender, histopathological classification, and anatomic site were plotted according to age at diagnosis in single years. ${ }^{16}$ Density plots showed smoothed age distributions using a non-parametric (model-free) approach. Two component mixture models considered the probability for a given melanoma case of being in either an early- or late-onset age distribution at diagnosis, assuming a flexible parametric model for the earlyand late-onset age distributions. ${ }^{17,} 18$ The key model parameter was the probability of being in the early-onset group.

Age-period-cohort (APC) models were used to simultaneously assess age, period, and cohort effects among men and women. ${ }^{19}$ Given the relationship [year of birth] = [year of diagnosis] minus [age at diagnosis], we had twenty-two partially overlapping 10-year birth-cohorts from 1890 to 2000 (referred to by the mid-year of birth), calculated from our six 5-year time periods and seventeen 5-year age groups. An important and identifiable APC parameter was the "fitted" age of onset curve, ${ }^{12}$ which we estimated for CMM in men and women separately. Specifically, the fitted curves provided estimates of the cross-sectional age-specific incidence rate curves, adjusted for potential period and cohort effects. For example, period effects could differ between males and females if screening trends varied by gender, whereas cohort effects might differ if the profile of CMM risk factors such as sun exposure changed differentially in successive cohorts of men and women. 


\section{RESULTS}

\section{Descriptive statistics}

SEER 9 collected $n=96,900$ invasive melanoma cases from 1975 to 2004 (table 1). Melanomas were $41 \%$ more common among men than women (male to female $I R R_{M F}=1.41$ ). Prior to age 40 years, age-specific incidence rates were lower for men than women ( $\operatorname{IRR}_{\mathrm{MF}}<1.0$ ), after which age-specific rates were higher for men (IRR $\left.\mathrm{MF}_{\mathrm{F}}>1.0\right)$. $\mathrm{IRR}_{\mathrm{MF}}$ were greater for males than females for all tumor characteristics, except for melanomas of the lower extremity $\left(I R R_{M F}=0.39\right)$. For all melanoma cases combined, incidence rates were approximately 5 -fold greater for superficial spreading melanoma (SSM) than for either nodular melanoma (NM) or lentigo maligna melanoma (LMM), i.e., 5.7 compared to 1.2 or 1.1 per 100,000 person-years, $p<0.001$ for difference. Acral melanomas (ACM) were rare (0.1 per 100,000 person-years). Unclassified melanomas or melanoma not otherwise specified (UCM) were at least as common as SSM. Melanomas were slightly more common in intermittently sun exposed than chronically sun exposed skin ( 7.6 versus 6.2 per 100,000 person-years). The most common anatomic sites were the trunk among men (6.8 per 100,000 person-years) and the lower extremity among women $(4.0$ per 100,000). Eighty percent of LMMs $(80.2 \%, 5,400$ of 6,735) compared to $36.7 \%$ of SSMs $(14,171$ of 38,574) occurred in sun exposed skin. Malignant melanomas were more likely to be thin $(\leq 1.0 \mathrm{~mm})$.

For all cases combined (total), incidence rates rose during the study period (table 2). Total rates were $84.4 \%$ (95\% CI, 80.9-88.0\%) higher in 1995-2004 than in 1975-84. Rates were greater for men than women for all time-periods, and this gender gap widened over time (IRR $\mathrm{MF}_{\mathrm{F}}$ rose from 1.2 during 1975-1984 to 1.5 during 1995-2004). Figure 1A also shows that age-adjusted incidence rates rose slightly faster (steeper slope) for men than women. As shown in table 2, the greatest percentage changes in incidence rates occurred among older persons (ages 70-79 years, 178\%), LMM (125\%), and chronically sun exposed skin (98.4\%).

\section{Age incidence patterns (rates and age distributions at diagnosis)}

Consistent with table 1, cross-sectional age-specific incidence rates were greater for women than men prior to age 40 years (figure 1B), after which rates were greater for men. Near age 40 years, there was a qualitative (crossover or reversing) age interaction by gender ( $p \approx 0$ for the null hypothesis of no age interaction by gender). Crossing age-specific incidence rates reflected bimodal age distributions at diagnosis (figure 2A). That is, the non parametric estimate of age distributions at diagnosis (density plots) showed a bimodal pattern of earlyand late-onset cancer populations in both men and women (figure 2A). However, males had a predominantly late-onset age distribution, whereas females had a predominantly early-onset distribution. Non parametric age distributions also were observed by histopathological classification (figure 2B) and anatomic body site (figure 2C). LMM and FHN had a predominantly late-onset age distribution, whereas SSM and LE had a predominantly earlyonset distribution. Consequently, the combination of male gender + LMM + FHN showed mostly a late-onset melanoma population (figure 2D), whereas the combination of female gender + SSM + LE resulted in a predominant early-onset age distribution at diagnosis.

In all cases considered, two component mixture models fit the data better than a single melanoma population. For example, among men during the calendar-period 1975-1984 (table 3), Akaike criteria (AIC) was larger for a mixture model (-39596) than for a single density $(-39614)$, consistent with a better fit for a mixture than for a single cancer population. For men, the proportion of cases corresponding to the earlier onset mode increased during the study period, with a greater probability of being in the earlier versus later group over time, i.e., the proportion of cases from the early age-at-onset distribution rose from $38.7 \%$ in $1975-1984$ to $46 \%$ in 1985-1994 to $75 \%$ in 1995-2004. Among women, AICs also were larger for a mixture 
than single cancer population, but women had a predominant early-onset age distributions for all time periods.

\section{Age period cohort (APC) models}

Period and cohort deviations differed between men and women $(p<0.01$ for the null hypothesis of no difference by gender). The APC "fitted" age-at-onset curves that were adjusted for period and cohort effects also were different for men and women (figure $3, p \approx 0$ for the null hypothesis of no difference by gender). Similar to the cross-sectional age-specific incidence rates (figure 1B), the APC fitted curves showed crossing or reversing age-specific incidence rates near age 40 years, consistent with qualitative age interaction. The age-specific incidence rates were greater for women than men prior to age 40 years, after which rates were greater for men.

\section{DISCUSSION}

More than 40 years ago, Mishima proposed two age-related pathways for cutaneous malignant melanomas CMMs that were linked to sun exposure (e.g., melanocytic and nevocytic). ${ }^{20}$

Chronic sun exposure was a direct risk factor for melanocytic but not for nevocytic melanomas. Melanocytic melanomas developed later in life, had slower rates of growth, metastasis, and invasion. Nevocytic melanomas occurred earlier in life, and had faster rates of proliferation. Following Mishima, Whiteman et al also suggested at least two melanomagenic pathways, one associated with chronic sun exposure and the other with aberrant nevi. ${ }^{21}$

Divergent CMM pathogenesis also appears to be embodied within the original histopathological classification schemes of Clark and McGovern. ${ }^{22-25}$ Chronic sun exposure is a risk factor for LMM (a.k.a., melanocytic) but less so for nevi-prone SSM (a.k.a., nevocytic). LMMs develop later in life, on sun exposed skin, and usually have slower rates of growth. SSMs occur earlier in life and commonly arise in relatively sun protected body sites. In fact, our population-based analysis confirmed predominantly late and early age distributions at diagnosis for LMM and SSM, respectively (figure 2B).

However, not all dermatopathologists and clinicians acknowledge the importance of melanoma histopathological classification. ${ }^{26}$ Unclassified melanomas accounted for $40.6 \%$ of CMMs in SEER (table 1, UCM $=39,378$ of 96,900). Also, melanoma subtype is not included in the current prognostic scheme of American Joint Committee on Cancer Staging system for CMM. 27 Therefore, the clinical relevance of histopathology for CMM may be somewhat uncertain and/or underestimated.

Nonetheless, recent molecular studies have linked CMM histopathology with somatic and germline genetic variants, sun exposure, and anatomic body site. CMMs arising among persons with chronic sun damaged skin are generally LMM, associated with somatic KIT mutations and p53 overexpression, and develop later in life. ${ }^{1,28,29}$ In contrast, CMMs occurring without sun-damaged skin are correlated with somatic $B R A F$ or $N-R A S$ mutations and germline $M C 1 R$ variants in some populations. ${ }^{30-33}$ These lesions typically develop earlier in life, among nevi-prone individuals, and are commonly SSM.

In this analysis, we used descriptive epidemiology and a structured mathematical approach to further characterize the relationship between aging and CMM incidence. Results revealed a robust (reproducible and reliable) age-interaction with early-onset and late-onset melanomas in the general population. Gender, histopathological classification, and anatomic site were all age-dependent effect modifiers for CMM risk. Women and men had relative excesses of earlyonset and late-onset CMMs, respectively. More specifically, early-onset cases were enriched by the combination of female gender, SSM, and LE; whereas late-onset patterns were enriched by the combination of male gender, LLM, and FHN (figure 2D). Lachiewicz et al recently 
demonstrated similar incidence patterns by gender and body site, using SEER data (2000 to 2004). ${ }^{34}$ Our analysis adds histopathology, a longer time frame (1975 to 2004), and a more comprehensive mathematical framework.

A strength of this study was the large-scale and population-based design of the SEER database that is generally representative of the United States population. Our descriptive observations also were supplemented with novel two-component mixture models and "comparative" ageperiod-cohort models. Limitations included non-standardized histopathological typing and missing data. Indeed, nearly $41 \%$ of CMMs were not classified in SEER. However, while the lack of complete histopathological classification was likely due to inconsistencies in reporting of melanoma attributes including classifying CMMs, our results still showed distinct incidence rate patterns for SSM and LMM. In fact, a systematic dermatopathological review would probably have enhanced our findings, which certainly reflected some 'real' world random variation among the many general pathologists contributing to SEER.

In sum, these data are consistent with a large body of established clinical and emerging molecular evidence demonstrating the etiologic heterogeneity of melanoma development and tumor progression, with a possible link between advancing age, solar exposure, and constitutive molecular changes. Early-onset CMMs may represent gene-sun exposure interactions occurring early (and/or intermittently) in life in more susceptible individuals and often arising from precursor lesions. The later onset melanomas may reflect the cumulative effects of lifelong (accumulated) sun exposure in somewhat less susceptible individuals. Indeed, divergent age-related cancer pathways suggest the aging is an effect modifier of melanomagenesis. Future analytical studies should be adequately powered to account for this age-dependent biological heterogeneity for acknowledged (gender, histopathology, and anatomic site) as well as suspected melanoma risk factors such as constituent genetic variants. Arguably, these large-scale and population-based results would have been difficult (if not impossible) to detect in smaller analytic studies; and therefore, new etiological clues can still be gleaned from descriptive and/or retrospective studies, especially when supplemented with a systematic mathematical approach.

\section{Acknowledgments}

This research was supported in part by the Intramural Research Program of the NIH/National Cancer Institute. We thank the reviewers for helpful comments, which improved the content of this manuscript.

\section{References}

1. Curtin JA, Busam K, Pinkel D, Bastian BC. Somatic activation of KIT in distinct subtypes of melanoma. J Clin Oncol 2006;24(26):4340-6. [PubMed: 16908931]

2. Fecher LA, Cummings SD, Keefe MJ, Alani RM. Toward a molecular classification of melanoma. J Clin Oncol 2007;25(12):1606-20. [PubMed: 17443002]

3. Elwood JM, Lee JA, Walter SD, Mo T, Green AE. Relationship of melanoma and other skin cancer mortality to latitude and ultraviolet radiation in the United States and Canada. Int J Epidemiol 1974;3 (4):325-32. [PubMed: 4435983]

4. Mack TM, Floderus B. Malignant melanoma risk by nativity, place of residence at diagnosis, and age at migration. Cancer Causes Control 1991;2(6):401-11. [PubMed: 1764565]

5. Elwood JM, Jopson J. Melanoma and sun exposure: an overview of published studies. Int J Cancer 1997;73(2):198-203. [PubMed: 9335442]

6. Hakansson N, Floderus B, Gustavsson P, Feychting M, Hallin N. Occupational sunlight exposure and cancer incidence among Swedish construction workers. Epidemiology 2001;12(5):552-7. [PubMed: 11505175]

7. Bulliard JL. Site-specific risk of cutaneous malignant melanoma and pattern of sun exposure in New Zealand. Int J Cancer 2000;85(5):627-32. [PubMed: 10699940] 
8. Fears TR, Scotto J, Schneiderman MA. Mathematical models of age and ultraviolet effects on the incidence of skin cancer among whites in the United States. Am J Epidemiol 1977;105(5):420-7. [PubMed: 860705]

9. Siskind V, Whiteman DC, Aitken JF, Martin NG, Green AC. An analysis of risk factors for cutaneous melanoma by anatomical site (Australia). Cancer Causes Control 2005;16(3):193-9. [PubMed: 15947871]

10. Tucker MA. Is sunlight important to melanoma causation? Cancer Epidemiol Biomarkers Prev 2008;17(3):467-8. [PubMed: 18319329]

11. Thompson WD. Effect modification and the limits of biological inference from epidemiologic data. J Clin Epidemiol 1991;44(3):221-32. [PubMed: 1999681]

12. Anderson WF, Rosenberg PS, Menashe I, Mitani A, Pfeiffer RM. Age-related crossover in breast cancer incidence rates between Black and White Ethnic Groups. J Natl Cancer Inst 2008;100(24): 1804-14. [PubMed: 19066264]

13. SEER-9. Surveillance, Epidemiology, and End Results (SEER) Program. (www.seer.cancer.gov) SEER *Stat Database: Incidence-SEER 9 Regs Limited-Use, Nov 2006 sub (1992-2004), National Cancer Institute, DCCPS, Surveillance Research Program, Cancer Statistics Branch, released April 2007, based on the November 2006 submission 2007

14. International Classification of Diseases for Oncology. US Interim Version 2000. Vol. 3. Geneva: World Health Organization; 2000.

15. Gail M, Simon R. Testing for qualitative interactions between treatment effects and patient subsets. Biometrics 1985;41(2):361-72. [PubMed: 4027319]

16. Anderson WF, Chatterjee N, Ershler WB, Brawley OW. Estrogen receptor breast cancer phenotypes in the Surveillance, Epidemiology, and End results database. Breast Cancer Res Treat 2002;76(1): 27-36. [PubMed: 12408373]

17. Anderson WF, Pfeiffer RM, Dores GM, Sherman ME. Comparison of age frequency distribution patterns for different histopathologic types of breast carcinoma. Cancer Epidemiol Biomarkers Prev 2006;15(10):1899-905. [PubMed: 17035397]

18. Pfeiffer RM, Carroll RJ, Wheeler W, Whitby D, Mbulaiteye S. Combining assays for estimating prevalence of human herpesvirus 8 infection using multivariate mixture models. Biostatistics 2008;9 (1):137-51. [PubMed: 17566074]

19. Holford TR. The estimation of age, period and cohort effects for vital rates. Biometrics 1983;39(2): 311-24. [PubMed: 6626659]

20. Mishima Y. Melanocytic and nevocytic malignant melanomas. Cellular and subcellular differentiation. Cancer 1967;20(5):632-49. [PubMed: 6024276]

21. Whiteman DC, Watt P, Purdie DM, Hughes MC, Hayward NK, Green AC. Melanocytic nevi, solar keratoses, and divergent pathways to cutaneous melanoma. J Natl Cancer Inst 2003;95(11):806-12. [PubMed: 12783935]

22. Clark WH Jr, From L, Bernardino EA, Mihm MC. The histogenesis and biologic behavior of primary human malignant melanomas of the skin. Cancer Res 1969;29(3):705-27. [PubMed: 5773814]

23. Clark WH Jr, Ainsworth AM, Bernardino EA, Yang CH, Mihm CM Jr, Reed RJ. The developmental biology of primary human malignant melanomas. Semin Oncol 1975;2(2):83-103. [PubMed: 790575]

24. McGovern VJ, Cochran AJ, Van der Esch EP, Little JH, MacLennan R. The classification of malignant melanoma, its histological reporting and registration: a revision of the 1972 Sydney classification. Pathology (Phila) 1986;18(1):12-21.

25. McGovern VJ. The classification of melanoma and its relationship with prognosis. Pathology (Phila) 1970;2(2):85-98.

26. Ackerman AB. Malignant melanoma: a unifying concept. Hum Pathol 1980;11(6):591-5. [PubMed: 7450735]

27. Balch CM, Buzaid AC, Soong SJ, Atkins MB, Cascinelli N, Coit DG, et al. Final version of the American Joint Committee on Cancer staging system for cutaneous melanoma. J Clin Oncol 2001;19 (16):3635-48. [PubMed: 11504745]

28. Whiteman DC, Parsons PG, Green AC. p53 expression and risk factors for cutaneous melanoma: a case-control study. Int J Cancer 1998;77(6):843-8. [PubMed: 9714052] 
29. Purdue MP, From L, Kahn HJ, Armstrong BK, Kricker A, Gallagher RP, et al. Etiologic factors associated with p53 immunostaining in cutaneousmalignant melanoma. Int J Cancer 2005;117(3): 486-93. [PubMed: 15900597]

30. Maldonado JL, Fridlyand J, Patel H, Jain AN, Busam K, Kageshita T, et al. Determinants of BRAF mutations in primary melanomas. J Natl Cancer Inst 2003;95(24):1878-90. [PubMed: 14679157]

31. Curtin JA, Fridlyand J, Kageshita T, Patel HN, Busam KJ, Kutzner H, et al. Distinct sets of genetic alterations in melanoma. N Engl J Med 2005;353(20):2135-47. [PubMed: 16291983]

32. Landi MT, Bauer J, Pfeiffer RM, Elder DE, Hulley B, Minghetti P, et al. MC1R germline variants confer risk for BRAF-mutant melanoma. Science 2006;313(5786):521-2. [PubMed: 16809487]

33. Cohen Y, Rosenbaum E, Begum S, Goldenberg D, Esche C, Lavie O, et al. Exon 15 BRAF mutations are uncommon in melanomas arising in nonsun-exposed sites. Clin Cancer Res 2004;10(10):34447. [PubMed: 15161700]

34. Lachiewicz AM, Berwick M, Wiggins CL, Thomas NE. Epidemiologic support for melanoma heterogeneity using the Surveillance, Epidemiology, and End Results Program. J Invest Dermatol 2008;128(1):243-5. [PubMed: 17713570] 

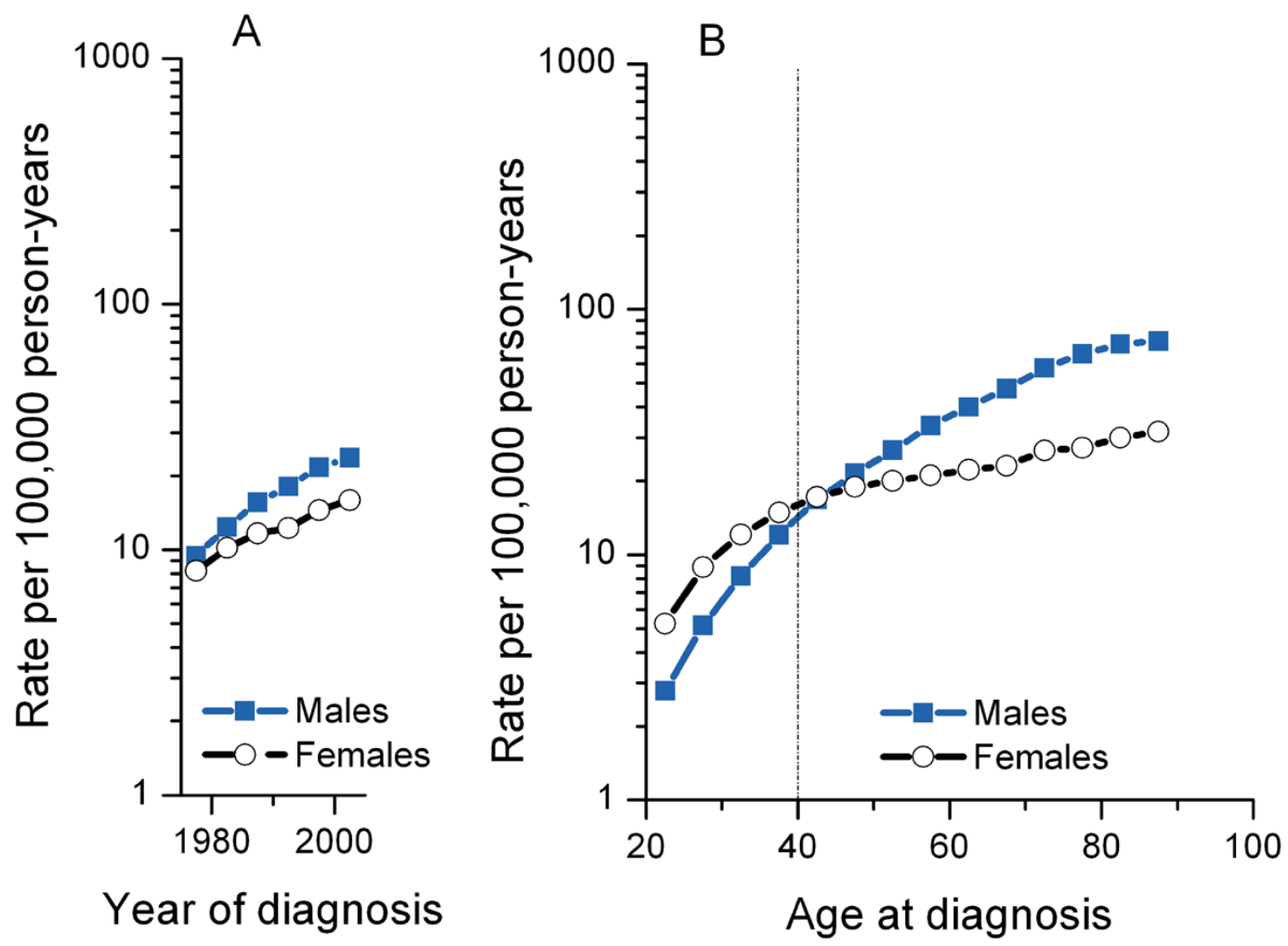

Figure 1.

Age-adjusted (2000 US standard population) incidence trends and age-specific incidence rates among men and women with cutaneous malignant melanoma (CMM), using the National Cancer Institute's Surveillance, Epidemiology, and End Results program (SEER 9, 19752004). 

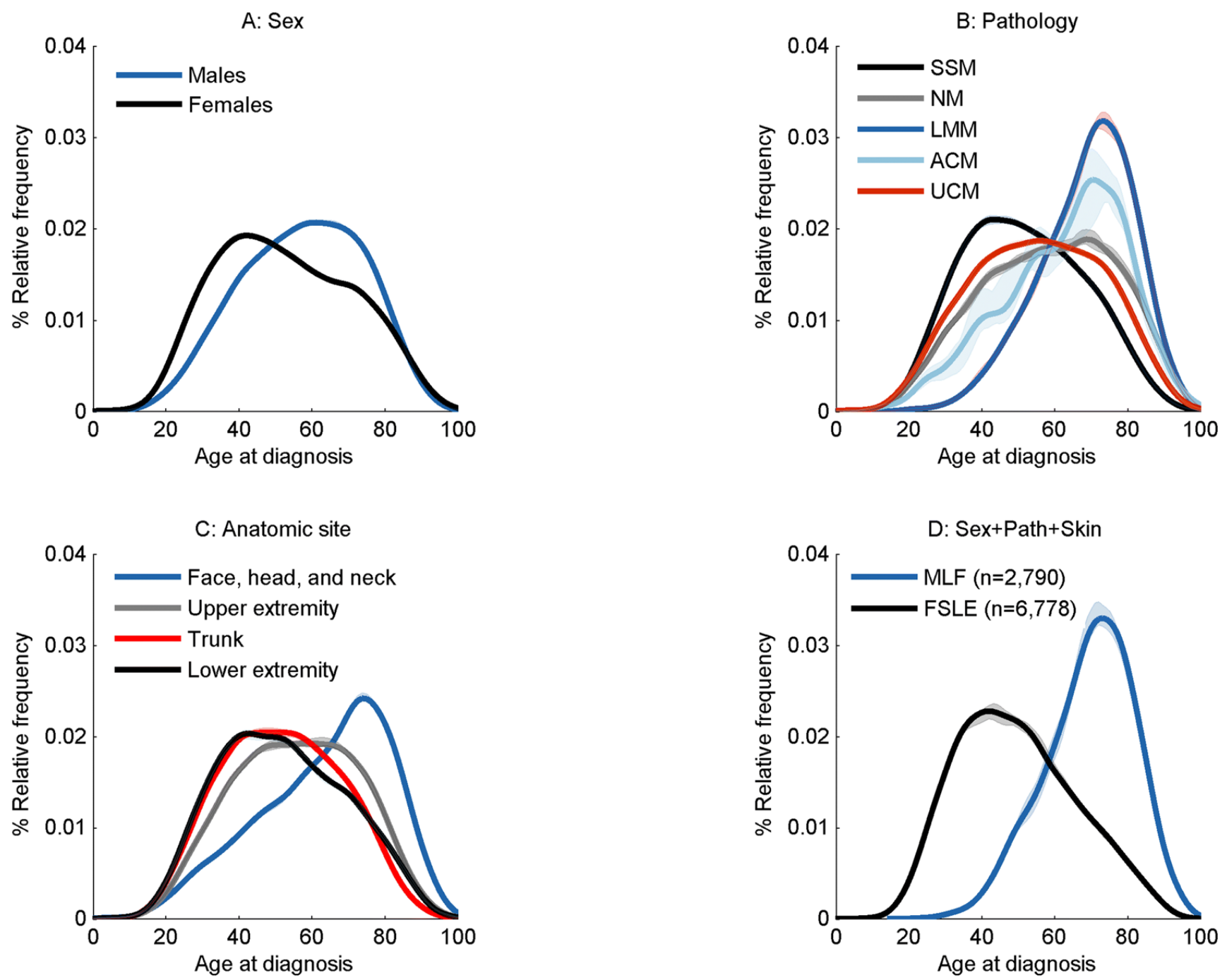

Figure 2.

Age distributions at diagnosis for gender, histopathology, and body site, using the National Cancer Institute's Surveillance, Epidemiology, and End Results program (SEER 9, 19752004). The area under each density plot represented $100 \%$ of melanoma cases, where density $\mathrm{X} 100=$ percent relative frequency distribution. Abbreviations: SSM, superficial spreading melanoma; LMM, lentigo maligna melanoma; ACM, acral melanoma; UCM, unclassified melanoma; MLF; men + LMM + face, head, and neck; FSLE, females + SSM + lower extremity. 


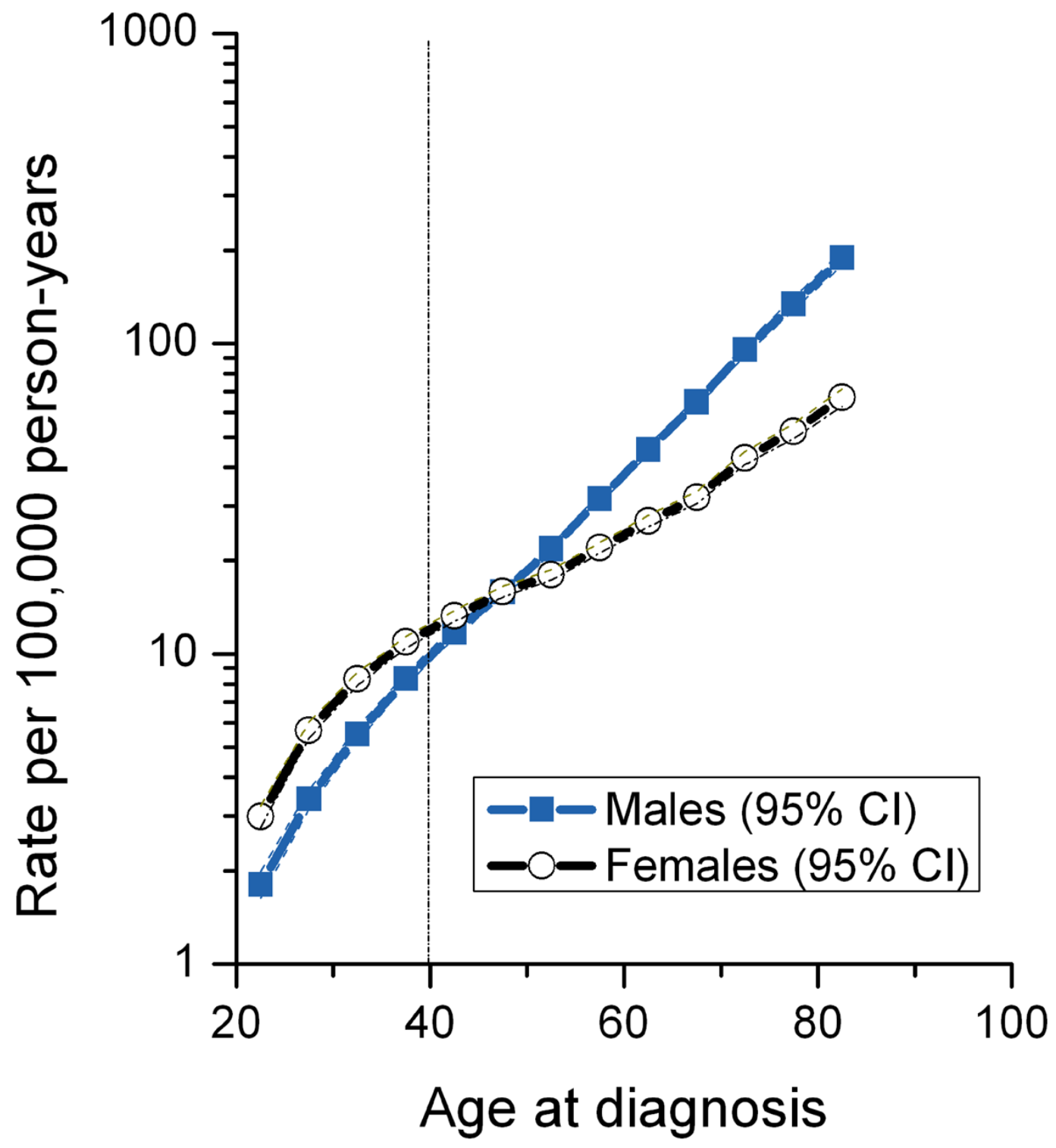

Figure 3.

APC "fitted" age-at-onset curve, adjusted for calendar-period and birth-cohort effects (SEER 9, 1975-2004). 
Anderson et al.

Page 12

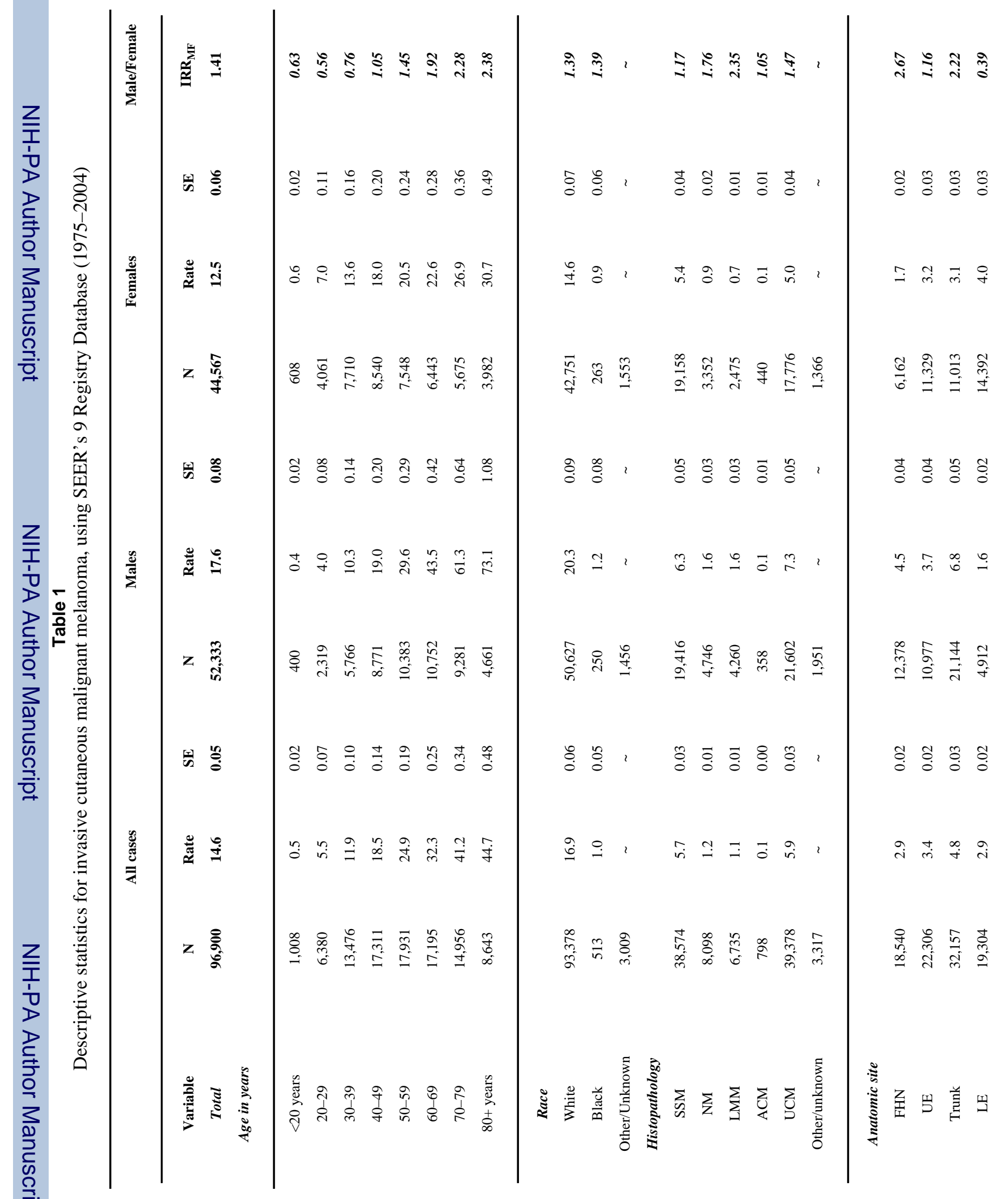


Anderson et al.

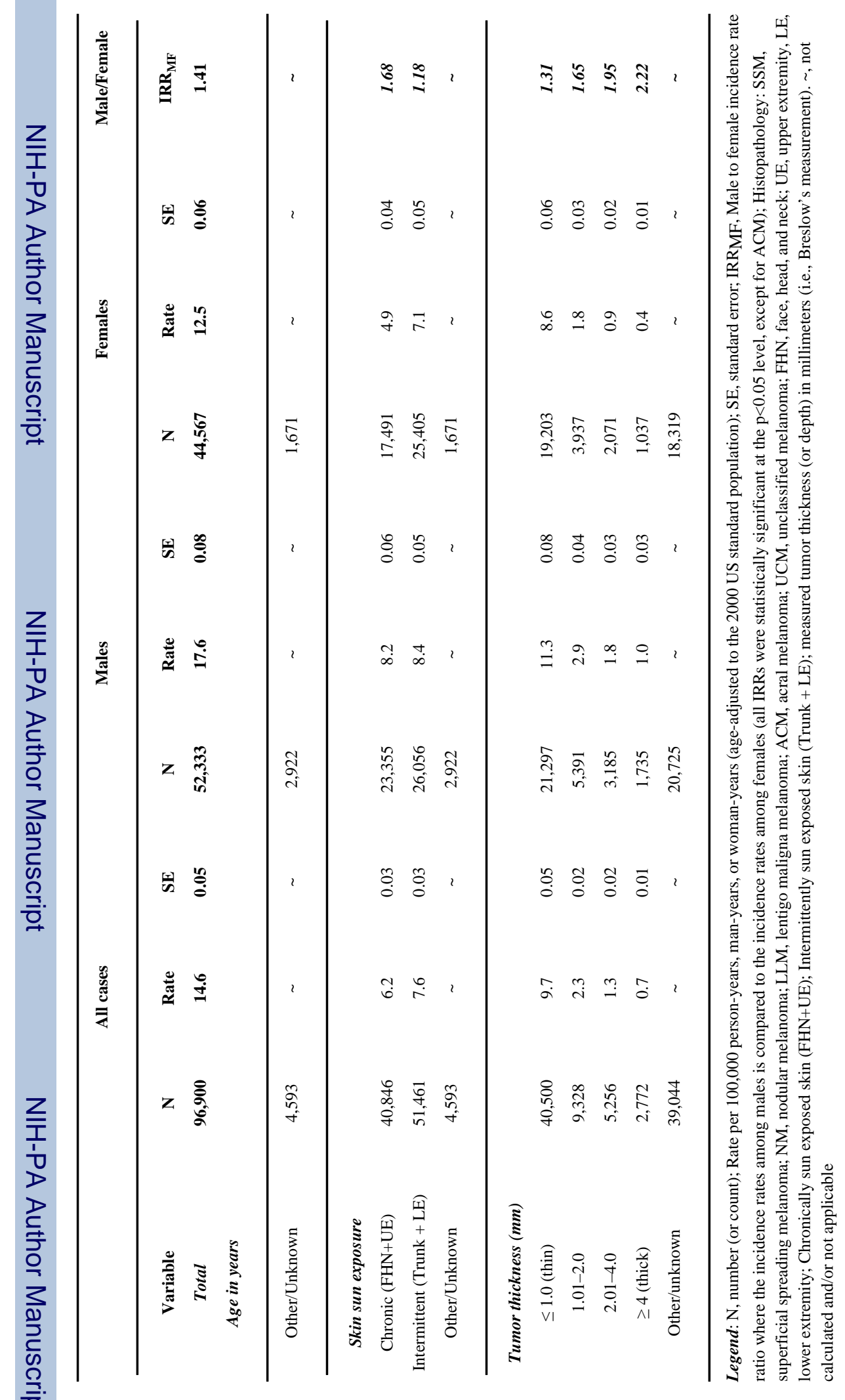




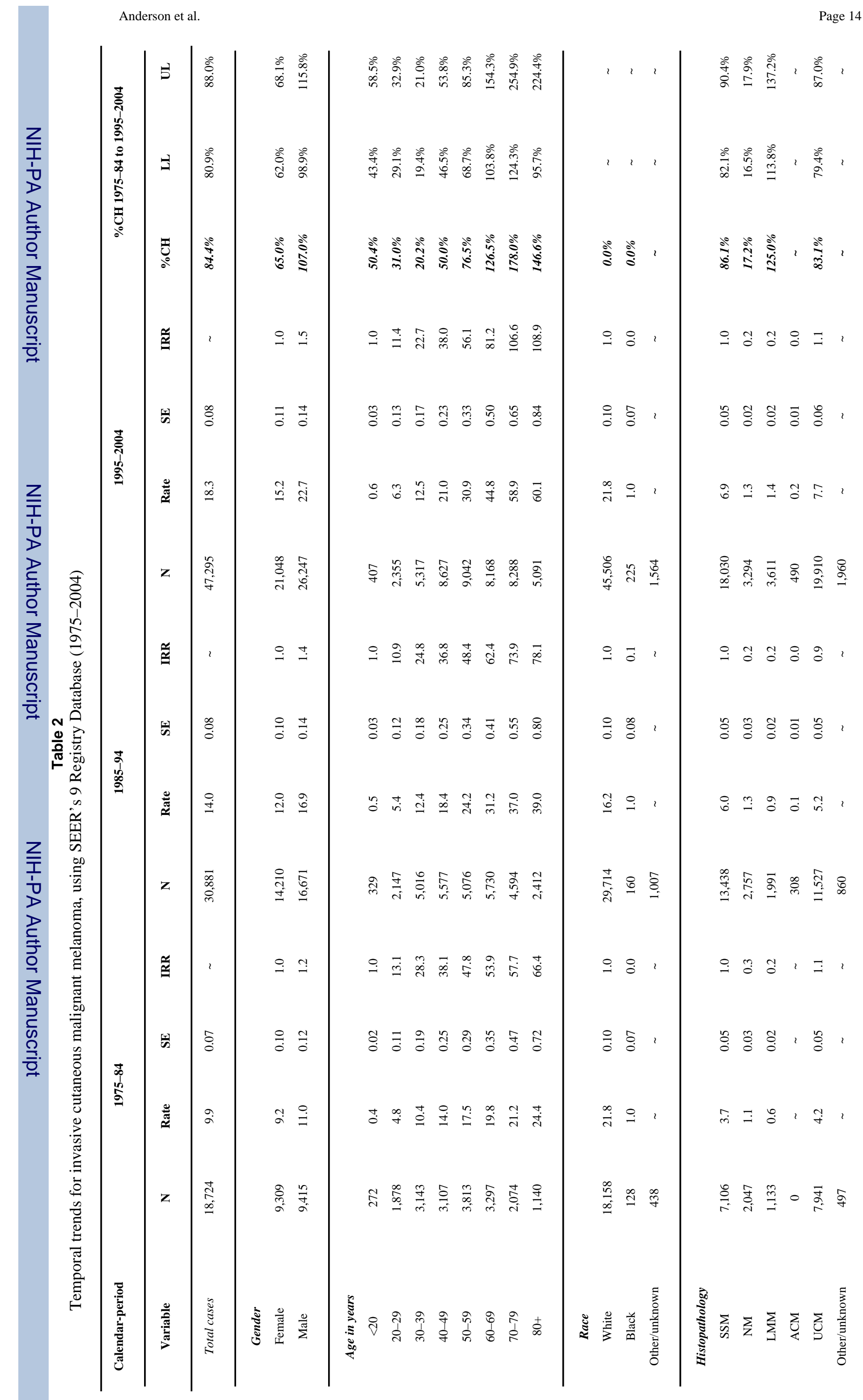




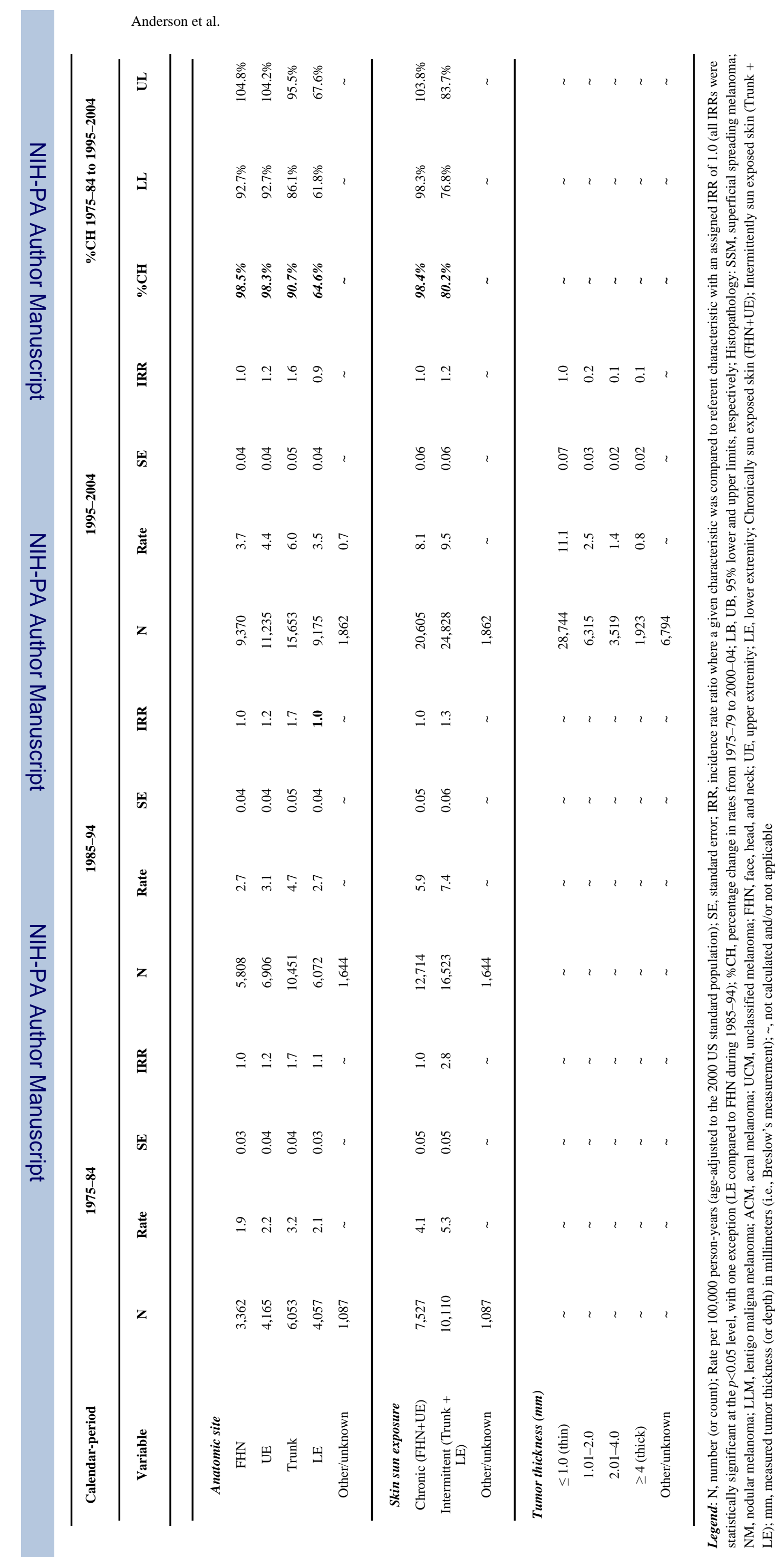




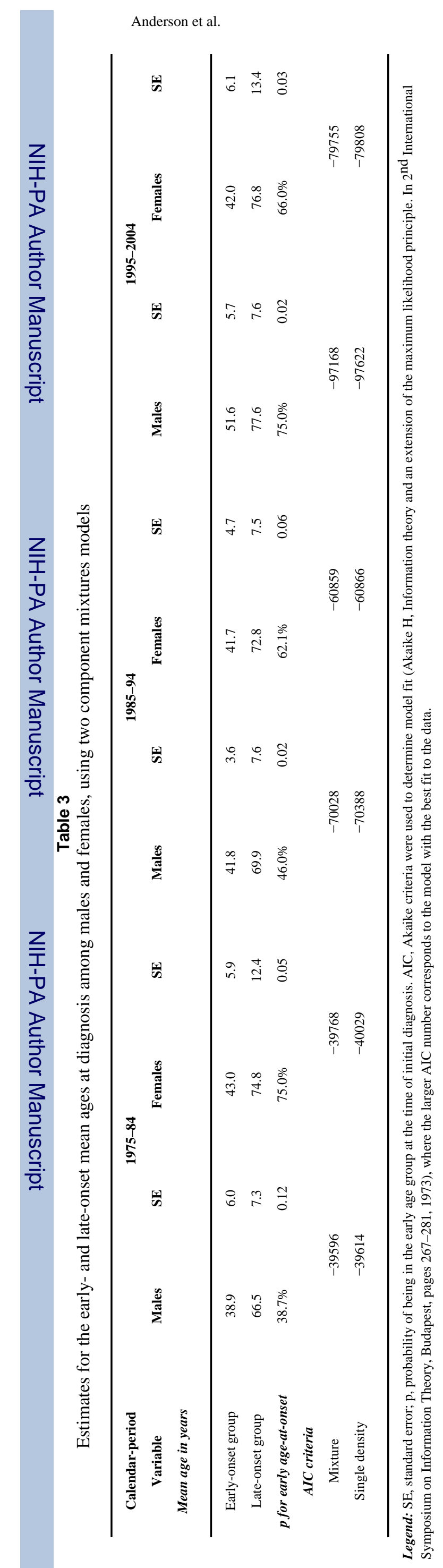

Page 16 